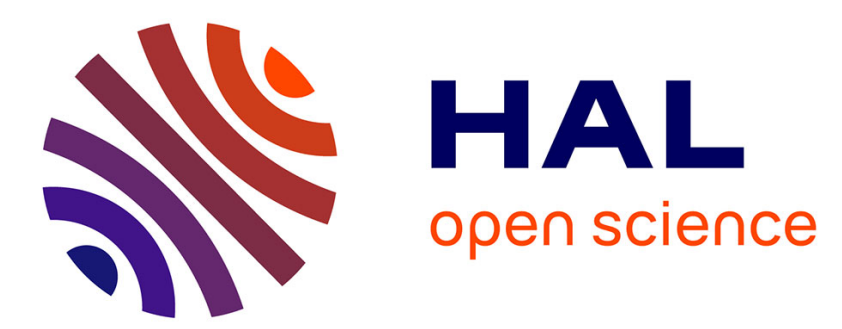

\title{
POUVOIRS LOCAUX ET CONTRAINTES JUDICIAIRES DANS UNE AFFAIRE DE VIOL EN INDE \\ Daniela Berti
}

\section{- To cite this version:}

Daniela Berti. POUVOIRS LOCAUX ET CONTRAINTES JUDICIAIRES DANS UNE AFFAIRE DE VIOL EN INDE. Diogène: Revue internationale des sciences humaines, 2012, 3, pp.139-165. 10.3917/dio.239.0139 . hal-02334470

\author{
HAL Id: hal-02334470 \\ https://hal.science/hal-02334470
}

Submitted on 29 Oct 2019

HAL is a multi-disciplinary open access archive for the deposit and dissemination of scientific research documents, whether they are published or not. The documents may come from teaching and research institutions in France or abroad, or from public or private research centers.
L'archive ouverte pluridisciplinaire HAL, est destinée au dépôt et à la diffusion de documents scientifiques de niveau recherche, publiés ou non, émanant des établissements d'enseignement et de recherche français ou étrangers, des laboratoires publics ou privés. 


\title{
POUVOIRS LOCAUX ET CONTRAINTES JUDICIAIRES DANS UNE AFFAIRE DE VIOL EN INDE
}

\author{
Daniela Berti
}

P.U.F. | Diogène

2012/3 - n० 239-240

pages 139 à 165

ISSN 0419-1633

Article disponible en ligne à l'adresse:

http://www.cairn.info/revue-diogene-2012-3-page-139.htm

Pour citer cet article :

Berti Daniela, «Pouvoirs locaux et contraintes judiciaires dans une affaire de viol en Inde »,

Diogène, 2012/3 n²39-240, p. 139-165. DOI : 10.3917/dio.239.0139

numéro spécial: "Les Frontières de la loi. Justice, pouvoirs et politique", dir. D.Berti \& G.Tarabout

Distribution électronique Cairn.info pour P.U.F..

(c) P.U.F.. Tous droits réservés pour tous pays.

La reproduction ou représentation de cet article, notamment par photocopie, n'est autorisée que dans les limites des conditions générales d'utilisation du site ou, le cas échéant, des conditions générales de la licence souscrite par votre établissement. Toute autre reproduction ou représentation, en tout ou partie, sous quelque forme et de quelque manière que ce soit, est interdite sauf accord préalable et écrit de l'éditeur, en dehors des cas prévus par la législation en vigueur en France. II est précisé que son stockage dans une base de données est également interdit. 


\title{
POUVOIRS LOCAUX ET CONTRAINTES JUDICIAIRES DANS UNE AFFAIRE DE VIOL EN INDE
}

\author{
par
}

DANIELA BERTI ${ }^{1}$

Un article de presse récemment publié en Inde et intitulé Police to videograph evidence annonce que la police de l'État de l'Himachal Pradesh (dans le nord du pays), dont les principaux commissariats de la région sont équipés de caméras digitales, sera dorénavant obligée d'inclure dans ses dossiers un enregistrement filmé des déclarations des témoins (The Tribune, Himachal Pradesh ed., 25 juillet 2011). L'article explique le but de cette mesure : éviter qu'au moment d'un procès les témoins du procureur, y compris les victimes, nient ce qu'ils ont dit à la police au moment de l'enquête et deviennent, selon le vocabulaire de la common law, des hostile witnesses (témoins à charge qui se rétractent). La rétractation des témoins lors d'un procès est un phénomène qui se produit régulièrement dans les tribunaux du subcontinent, dans des cas importants et médiatisés comme dans la routine des affaires judiciaires. Elle provoque souvent chez les procureurs et chez les juges une attitude de résignation et d'impuissance.

Dans une pratique de la justice marquée par une prolifération de l'écrit, la mise en avant de l'enregistrement vidéo comme support de la preuve judiciaire, tout en témoignant d'une dévalorisation de l'écriture, est également le signe d'une certaine défiance envers l'oralité. Elle met en question le principe même sur lequel repose le système de la common law, selon lequel la vérité judiciaire doit être atteinte par le témoignage oral que le témoin donne sur le vif au moment du procès (Davies, Croall \& Tyrer 1995). Ce principe d'oralité, prescrit par un code de procédure pénale qui a été introduit en Inde par les Britanniques, contraste avec l'idée constamment réitérée par les juges et les procureurs, selon laquelle la plupart des témoins qui sont appelés à soutenir lors du procès le chef d'accusation nient ce qu'ils auraient auparavant déclaré aux policiers. Il semble aussi, d'une certaine façon, être contredit par une prédominance de l'écrit qui caractérise la procédure au pénal, laquelle prévoit la longue et méticuleuse traduction et transcrip-

1. Cet article est issu du programme de recherche ANR (08-GOUV-064) " Justice et Gouvernance en Inde et en Asie du Sud " (www.just-india.net).

Diogène n² 239-240, juillet-octobre 2012. 
tion écrite de ce qui est dit au moment de l'audience (Berti 2010, 2011a).

Dans cette contribution, j'analyserai ce rapport contrasté entre écriture et oralité dans la production de la preuve judiciaire, en particulier dans sa relation au problème posé par les hostile witnesses. Je m'appuierai pour cela sur une affaire criminelle observée dans un tribunal de district, concernant le viol d'une mineure, pour analyser comment les procédures judiciaires arrivent à être perturbées par des dynamiques extrajudiciaires de pouvoir liées aux rapports locaux de domination. C'est là un point crucial d'une question plus générale, que les auteurs qui s'intéressent au fonctionnement des tribunaux en Inde se sont à plusieurs reprises posée. Comment un système de justice hérité de la société coloniale, et centré sur un régime de preuve et de vérité né ailleurs, peut-il fonctionner dans une société qui reste largement fondée sur des rapports de hiérarchie et de domination locale centrés sur la religion, le genre, le statut de caste, la dépendance économique, ainsi que sur des allégeances féodales, familiales ou territoriales? Comment la justice de l'État, indépendante et séculière, peut-elle fonctionner face à ces multiples sphères d'autorité hautement coercitives, notamment en milieu rural ?

Les premiers auteurs à s'être posé cette question, à partir des années 1960, font référence à un "clash de valeurs" entre alien state et société indigène (Cohn 1959), ou entre jurisprudence légale et jurisprudence locale (Cohn 1965). Deux types de considérations en ont découlé. D'une part, la difficulté à appliquer la justice de l'État auprès de la communauté villageoise a pu être analysée, dans la perspective des subaltern studies, en termes de "résistance" à l'autorité coloniale et postcoloniale ${ }^{2}$. De l'autre, le fait que la justice d'État pouvait au contraire parfois être préférée par les villageois, en fonction de l'intérêt personnel que ceux-ci pouvaient en tirer, a été interprété en termes de "manipulation" ou de "calcul stratégique» (Cohn 1959). Les deux attitudes étaient d'ailleurs compatibles, comme le montre la notion de bilégalité introduite par l'anthropologue M. N. Srinivas et reprise ensuite par plusieurs auteurs (Galanter 1992, Shah 1998) ${ }^{3}$.

La spécificité coloniale, ou postcoloniale, de la dissonance entre un système de justice dicté par l'État et les multiples formes

2. Brown (2003), Baxi (2003), Guha (1989). Pour une analyse de l'histoire de ces théories, voir Wardhaugh (2005), Ludden (2001), Sivaramakrishnan (2008). L'idée de résistance est aussi impliquée par l'idée d'utilisation de la loi comme " arme des faibles" (Eckert 2006).

3. Le terme "bilégal" renvoie au fait que les villageois peuvent utiliser aussi bien le système indigène que la loi officielle, selon l'estimation qu'ils font de leur intérêt (Srinivas 1962). 
d'autorité locale a été critiquée par Anderson (1990) qui, s'appuyant sur des travaux historiographiques portant sur la période médiévale, mit en avant le fait qu'un tel écart existait déjà à la période précoloniale, dans les rapports entre royaumes et d'autres formes de pouvoir plus localisées (Stein 1980). Par ailleurs, l'opposition dyadique entre " indigène " et " étranger " présupposerait, selon cet auteur, "une uniformité socioculturelle des deux côtés de la dichotomie qui est probablement inexistante" (Anderson 1990 : 172).

D'autres travaux dédiés à la question de la mise en œuvre de la justice en Inde ont fait ressortir la disparité entre les "promesses " des réformes légales (Anderson 1990: 175) visant à mettre fin à différents types d'inégalités sociales, et le dysfonctionnement du système judiciaire, ou de ses fonctionnaires, qui empêcherait la réalisation de ces promesses et qui reproduirait, dans sa pratique, les mêmes rapports de domination qu'il déclare vouloir déraciner.

Une approche qui évite de tomber dans le "piège dualiste" de l'opposition entre État étranger (alien state) et pratiques locales, ou entre hégémonique et subalterne, est celle qui a été adoptée, en dehors du contexte indianiste, par Merry (2003) dans son étude sur les victimes de violences domestiques parmi les classes populaires nord-américaines. Dans ce travail, l'auteur se pose la question de comprendre comment telle ou telle personne en vient à choisir de s'adresser à la justice de l'État au lieu de rester enfermée dans des dynamiques de domination centrées sur la famille, la religion, la communauté. Comment une personne arrive-t-elle à comprendre son propre problème en termes de droits et à se voir comme un sujet porteur de droits (rights-bearing subject)? En se fondant sur plusieurs études de cas, Merry montre comment le fait de se percevoir comme un sujet de droit dépend d'une expérience positive du système judiciaire (police, procureurs, juges). L'auteur se réfère aussi à la notion de "subjectivités multiples " et potentiellement contradictoires, introduite par Moore (1994), selon laquelle chaque individu "assume des positions multiples en tant que sujet, à l'intérieur d'un ensemble de discours et de pratiques sociales, si bien qu'un sujet n'équivaut pas à un individu " (cité dans Merry $2003: 349$ ).

Cette attention portée aux multiples positions subjectives de personnes appartenant à un même milieu social marqué par une situation d'oppression permet de faire ressortir un cadre plus nuancé et plus diversifié du rapport entre justice d'État et dynamiques locales de domination. Elle peut d'ailleurs s'appliquer non seulement aux victimes mais aussi aux représentants de la machine judiciaire (juges, procureurs, policiers), avec lesquels celles-ci se trouvent interagir. C'est dans cette perspective que le cas présenté ici sera analysé. Il mettra en scène des protagonistes - que ce soit 
du côté du système judiciaire ou du côté des parties civiles - dont les comportements ne sont ni figés, ni dépourvus d'ambiguïté, et qui, selon les moments, recherchent l'affirmation de la loi ou, au contraire, reproduisent les rapports de domination villageois.

Dans un premier temps, je parcourrai brièvement les étapes principales de la procédure pénale indienne, en montrant en particulier comment ce rapport ambigu entre oral et écrit se manifeste dans le cas des témoins à charge qui se rétractent, les hostile witnesses. J'entrerai ensuite dans le détail d'une affaire afin de voir comment le déroulement du procès est déterminé par les dynamiques sociales du cas concerné.

\section{De l'oral et de l'écrit}

Une première question que l'on peut se poser lorsque l'on étudie une affaire criminelle en Inde consiste à se demander comment le crime est décrit (ou nié) par les parties impliquées. L'enregistrement d'une affaire par la police induit dans l'entourage de la victime et de l'accusé une multiplicité de récits rapportant des points de vue multiples et contrastés, des opinions, rumeurs, révélations. À la différence de l'ethnologue, pour qui ces récits seraient tous légitimes afin de mener son étude de cas, la spécificité de la décision judiciaire fait que ces sources d'informations ne peuvent être prises en compte par le juge que si elles ont été introduites par le biais de la procédure - que ce soit dans leur forme écrite, en tant que plaintes ou rapports de police, ou dans leur forme orale, à travers les déclarations des témoins aux audiences.

La valeur assignée à l'écriture et à l'oralité lors de la production de ces récits varie selon le déroulement de l'affaire. Ainsi, les auditions des témoins qui sont rédigées par la police sous forme de récits n'ont pas valeur de preuve car, comme on l'a vu, ce qui a été transcrit au moment de l'enquête doit être oralement confirmé par le témoin au moment du procès. Ce que ce dernier dit lors des audiences en réponse aux questions qui lui sont posées sera ensuite mis par écrit (toujours sous forme de récit) pour que les juges des cours supérieures puissent considérer cette déposition en cas d'appel. Comme le remarque Roussel (2005 : 23) pour la procédure inquisitoire suivie en France, ici aussi, et encore plus, le procès repose "sur un incessant va-et-vient entre l'oral et l'écrit, dans de subtils jeux d'infirmations et de confirmations, d'interférences et de références. " Mais de quel genre de récits s'agit-il ?

Si l'on considère uniquement les documents écrits, la première version du récit à être incluse dans les pièces du dossier correspond au First Information Report (FIR). Le FIR est rédigé par la police à la suite d'une plainte (orale ou écrite) ou directement par un officier de police. Il se présente sous la forme d'une narration écrite à 
la première personne par le plaignant, bien qu'il soit toujours rédigé par la police. Le récit des faits se présente déjà comme une version juridiquement formatée, répondant à des critères précis de la procédure judiciaire. Lorsque le FIR est enregistré à la suite d'une plainte écrite (de la victime ou de son représentant), celle-ci est aussi incluse dans le dossier. La plainte éventuelle précède donc la version du récit rapportée dans le FIR, mais elle n'assumera de valeur d'un point de vue judiciaire que si elle arrive à déclencher l'enregistrement de l'affaire par la police.

Une fois l'enquête commencée, les dépositions des témoins vont éclaircir d'autres aspects du récit. Les transcriptions de ces auditions sont rédigées par la police et forment des récits racontés à la première personne par le témoin, bien qu'ils transcrivent une interaction qui s'est effectuée sous forme de questions-réponses. Ces transcriptions ne sont pas signées par les témoins. Sans constituer des "preuves", elles serviront néanmoins comme "socle de l'accusation" (Roussel 2005 : 17) à partir duquel les questions qui seront adressées aux témoins lors du procès seront formulées. Ces transcriptions sont régulièrement mises en cause par les témoins, qui nient au moment du procès avoir dit ce que la police a écrit.

Parmi les documents initiaux du dossier judiciaire figure aussi le police diary, les notes de terrain rédigées au jour le jour par la police durant l'enquête. Ces notes incluent les interrogatoires de l'accusé menés lors de sa garde à vue. La transcription des paroles de l'inculpé par la police, contrairement aux transcriptions des interrogations des témoins, ne sera guère prise en compte lors du procès. Par contraste avec ce qui se passe en France, où l'aveu est toujours recherché (Roussel 2005 : 19), le tribunal en Inde s'attend à ce que l'accusé, souvent sur instruction de son avocat, se déclare innocent et nie les faits. Il arrive que dans le rapport de police l'accusé ait partiellement avoué les faits, mais que, lorsqu'il comparaît devant le juge, il nie toutes les accusations sans que personne ne tienne compte de ce que la police a écrit dans son rapport. Sa parole au cours du procès ne sera d'ailleurs pas sollicitée, sinon lorsque tous les témoins auront été entendus: on le confronte alors, de façon très procédurale, à une longue liste d'accusations (cf. infra).

Le début du procès reste dans le dossier sous forme de ce que l'on appelle evidence (preuves), c'est-à-dire les transcriptions des dépositions faites par les témoins au moment des audiences. Les evidence marquent le passage du dossier du hindi à l'anglais ${ }^{4}$. Au

4. L'usage de traduire et transcrire les dépositions des témoins en anglais est suivi dans la plupart des États de la Fédération. Il y a néanmoins quelques exceptions comme dans l'État du Penjab où les dépositions sont transcrites en punjabi. 
cours des audiences, au fur et à mesure que le témoin répond (en hindi) aux questions qui lui sont posées, le juge résume le dialogue en anglais en le transformant en récit fait à la première personne par le témoin. Les récits transcrits dans les evidence sont les plus consultés du dossier. Ils sont relus et cités par le juge pour rédiger son jugement, et par les juges des cours supérieures en cas d'appel.

Par rapport aux récits évoqués jusqu'à présent, ceux des evidence présentent un élément nouveau. Ils ne se limitent pas à transformer un dialogue en narration, mais indiquent aussi ce qui dans cette narration est considéré comme faux par la partie qui interroge. Ce passage du "présumé vrai " au "présumé faux " est marqué, dans le récit, par ce qui s'appelle en common law la crossexamination, qui correspond aux questions posées au témoin par la partie adverse (par exemple par l'avocat de la défense aux témoins du procureur). À la différence de l'examination-in-chief, au cours de laquelle le témoin est libre de répondre à la question qui lui est posée, dans la cross-examination il ne peut que confirmer ou nier ce qui est suggéré dans la question (leading questions) : il doit répondre oui ou non. Ces questions sont davantage utilisées pour que celui qui pose la question puisse insinuer une affirmation que pour obtenir une réponse, vu que souvent celui qui interroge sait déjà si le témoin confirmera ou niera ce qui est suggéré par la question. $\mathrm{Au}$ cours de la transcription des audiences, les leading questions sont très importantes car elles sont non seulement prononcées mais aussi traduites en anglais et transformées en récit, à la première personne, fait par le témoin. Contrairement à l'examinationin-chief, ici chaque phrase du récit est introduite par la formule "it is incorrect (or correct) that " ou "it is not true (or it is true) that " ce qui signifie que le témoin nie ou approuve ce que la question insinue. Le résultat peut être un récit où, paradoxalement, celui qui narre nie au fur et à mesure ce qui est dit (Berti 2011b).

Si la cross-examination des témoins par la partie adverse participe du déroulement ordinaire d'un procès, la cross-examination effectuée par la partie qui a appelé le témoin à la barre peut apparaître comme une perturbation du cours du procès, et la crédibilité du témoin commence à être mise en doute. C'est ce qui arrive très souvent avec les témoins du procureur lorsqu'ils sont interrogés par celui-ci ou par le juge lui-même : ils commencent par nier ce qu'ils sont supposés avoir dit à la police au moment de l'enquête. Les interactions commencent alors à devenir tendues. Le témoin est accusé (au nom du procureur) de ne pas dire la vérité, bien qu'il s'agisse d'une accusation "procédurale", faite pour être signalée au niveau de l'écrit, dans la transcription de l'audience. C'est là que le juge interrompt la linéarité du récit qu'il dicte à partir des dialogues auxquels il assiste. Il fait alors écrire par son greffier la phrase suivante : 
At this stage Learned Public Prosecutor has stated that witness is trying to suppress the truth. As such prayer is made to cross examine the witness, which is considered and allowed.

Cette formule est dictée directement en anglais sans que le témoin, qui en général ne parle que le hindi ou le dialecte de sa région, puisse la comprendre. Elle est souvent prononcée directement par le juge, tout en étant toujours présentée comme une demande provenant du procureur. C'est d'ailleurs le juge qui pose la plupart des questions au témoin, surtout s'il voit que le procureur n'a pas suffisamment préparé le cas ou n'est pas suffisamment combatif pour affronter un témoin réticent. Incité par les cours supérieures à ne pas rester un spectateur passif du procès, le juge de district intervient d'ailleurs souvent dans les interactions en interrogeant directement les témoins, que ce soit lors de l'examination-in-chief ou lors de la cross-examination. Seul à devoir décider - le système du jury a été aboli en Inde en 1960 - il n'a pas besoin d'expliquer les aspects techniques de la procédure. Lorsqu'il dicte en anglais au greffier que le témoin va maintenant être soumis à une crossexamination, il n'explique pas à celui-ci qu'à partir de là la véracité de ses paroles est mise en doute et que les questions serviront désormais à démontrer son manque de crédibilité.

À la fin des evidence, les faits sont à nouveau narrés au cours du statement of the accused prévu par la section 313 du Indian Evidence Act. Les accusations apparues au cours des audiences sont soumises à l'accusé pour qu'il puisse s'exprimer. Cette partie du procès ne réserve pas de surprises. Les questions sont préparées à l'avance sur la base de la transcription des audiences. "It has come in the prosecution evidence led against you that [...] What have you to say? " À chaque question l'accusé répond " it is incorrect ", ou bien " it is wrong ", ou encore "I don't know ", de façon presque automatique $^{5}$. Le "statement of the accused" se présente donc la plupart du temps comme une longue série de questions, indiquées comme telles dans la transcription, toujours suivies par le même genre de réponse. C'est seulement en fin de liste que la réponse peut parfois inclure des informations supplémentaires, lorsque l'on demande à l'accusé "Why this case has been registered against you ?" Le témoin répète alors ce que son avocat peut avoir déjà évoqué plusieurs fois au cours de la cross-examination - par exemple que l'affaire a été fabriquée par la police ou par un ennemi personnel.

5. La répétition des accusations est faite même lorsque le juge voit que l'accusation ne peut être prouvée par le procureur : c'est une obligation procédurale. Les questions sont alors souvent lues par le reader ou directement transcrites par le greffier en présence du procureur et de l'avocat. 
L'étape suivante du procès correspond aux arguments (plaidoirie), lors desquels le procureur et l'avocat défendent leur position devant le juge. Ici les evidence sont relues et argumentées en se référant à des précédents, mais rien n'est retranscrit. Le juge reprend les faits selon la succession des témoins qui se sont succédé lors du procès, ainsi que les arguments de l'accusation et de la défense puis, s'appuyant lui aussi sur des précédents, il tranche le cas en donnant sa décision ${ }^{6}$.

Cette multiplicité de récits appelle deux remarques principales. Il faut noter d'abord l'importance qu'il y a à transformer l'oralité en écriture judiciaire. Car si, d'une part, en conformité avec les procédures de la common law, l'oralité garde son importance par rapport à l'écrit, de l'autre, tout ce qui est dit est transformé par un procès d'écriture qui met des questions-réponses sous forme d'un récit à la première personne et qui traduit le hindi parlé en anglais juridique. Deuxièmement, il faut remarquer l'importance des questions par rapport aux réponses. Dans ce contexte d'interaction, le but des questions consiste moins à obtenir une réponse qu'à expliciter une contre-narration qui sera figée dans la version anglaise écrite, la version officielle.

Tenant compte de cette multiplicité de récits, j'analyserai maintenant un cas particulier, pour montrer comment cette tension entre l'oral et l'écrit n'est pas le seul fait de la transcription officielle d'une interaction orale avec un officier ou un professionnel de la justice. Elle a également trait à l'évolution de l'affaire pour les parties impliquées, aux négociations, pressions et propositions faites au niveau local et en dehors du tribunal. Pour comprendre ce qui se passe lors du procès, il faudra tenir compte non seulement des versions officielles incluses dans le dossier, mais aussi des discours que les gens impliqués dans l'affaire - les parties civiles, les témoins ou les professionnels - peuvent tenir à l'extérieur de la salle d'audience.

\section{Un double crime}

L'affaire présentée ici correspond à un cas de figure considéré comme plutôt fréquent en Inde : l'exploitation sexuelle d'une femme de basse caste par un homme de haute caste. L'accusé est Dhiman Sharma ${ }^{7}$, un jeune homme appartenant à la caste Brahmane, âgé d'une vingtaine d'années, d'un village isolé dans les montagnes à 3 ou 4 heures de route de Shimla, la capitale de l'État de l'Hima-

6. Ce travail minutieux de dictée et de transcription prend tout son sens en prévision d'un appel, à l'occasion duquel les faits seront à nouveau, et à plusieurs reprises, racontés.

7. Les noms de tous les protagonistes de l'affaire ont été modifiés afin de préserver leur anonymat. 
chal Pradesh. La victime, Anita, est une fillette du même village, âgée de 11 ans au moment du procès (en 2010), de caste Koli, une caste de très bas statut faisant partie des Scheduled Castes, les castes répertoriées, catégorie administrative s'appliquant à des castes autrefois dites " intouchables » qui bénéficient d'une protection en vertu de la Constitution indienne et de nouvelles lois interdisant la discrimination. Anita vivait avec sa mère et son petit frère chez les parents du garçon, pour la famille de qui sa mère travaillait comme servante.

Le First Information Report (FIR), rédigé par la police en juillet 2009, concerne trois sections du code pénal indien: la 376, qui condamne le viol " à la prison à vie ou à plus de 10 ans de prison "; la 354 , concernant "l'usage de la violence ou agression sur une femme avec l'intention d'outrager sa modestie "; et la section 506 contre "l'intimidation criminelle."

Selon le Indian Penal Code 8 le viol est une offense "cognizable ", c'est-à-dire que l'accusé peut être immédiatement arrêté, sans qu'une libération sous caution puisse lui être accordée. Il s'agit également d'une offense "non compoundable ": elle ne peut être résolue par un compromis privé entre les parties concernées, car elle est considérée comme une offense commise aussi contre la société. Aucun retrait de plainte n'est donc possible et le procès doit se dérouler jusqu'au verdict d'un juge du tribunal de district (Session Judge). De plus, l'affaire était enregistrée sous la section 3 du Scheduled Castes and Scheduled Tribes Prevention of Atrocities Act (à partir d'ici Sc/St Act), promulgué en 1989 par le Parlement afin de prévenir les "atrocités et les discriminations contre les castes de bas statut." Plus exactement, la section du Sc/St Act utilisée pour cette affaire condamne :

celui qui, n'étant pas un membre d'une Scheduled Caste ou d'une Scheduled Tribe, étant dans une position de dominer la volonté d'une femme appartenant à une Scheduled Caste ou à une Scheduled Tribe, utilise cette position pour l'exploiter sexuellement d'une façon qu'elle n'aurait pas acceptée autrement. (Sc/St Act, Ch. 2/1, xii.)

L'enregistrement d'une affaire de viol sous le Sc/St Act entraîne plusieurs contraintes procédurales, parmi lesquelles le fait que le policier autorisé à conduire l'enquête doit être un officier de rang au moins égal à Assistant Superintendent of Police. Cette mesure prévue par la loi est destinée à garantir que ce genre d'affaire, définie comme "très sensible", soit traité de façon appropriée ${ }^{9}$. Dans

8. Le Code de procédure pénale indien fait partie du projet de codification initié par les Britanniques en Inde dès la première moitié du XIXe siècle (Kolsky 2005).

9. Ces cas sont aussi censés être jugés par un tribunal spécial, même si c'est souvent le Session Judge qui s'occupe de l'affaire. 
ce cas, en outre, le suspect (toujours appelé en Inde " the accused" tout en étant considéré innocent du point de vue de la procédure) est alors présumé avoir commis le crime dont on l'accuse, et c'est à lui de fournir la preuve de son innocence.

Ces dispositions sont perçues comme très contraignantes par les castes considérées de haut statut. C'est ce qui explique le discours couramment mis en avant dans ces milieux, selon lequel le $S c / S t$ Act serait souvent détourné et manipulé par les castes inférieures, facilement tentées de faussement impliquer un membre des castes supérieures dans une affaire judiciaire.

Néanmoins, au sein des castes répertoriées, seule une partie de la population est au courant de l'existence de cette loi - dont le texte intégral n'existe qu'en anglais. Le degré de connaissance que les villageois de ces castes peuvent avoir de leurs droits dépend alors de plusieurs facteurs, parmi lesquels la présence dans la région de leaders politiques appartenant aux Scheduled Castes. Ceux-ci évoquent de telles questions légales pendant leurs discours publics ou dans les journaux locaux. Certains de ces leaders se définissent aussi comme des " activistes sociaux" et, ayant acquis une certaine compétence par rapport aux procédures judiciaires, peuvent aider les membres des Scheduled Castes à recourir aux lois que l'État a promulguées en leur faveur.

C'est ce qui s'est passé dans le cas présent. L'enregistrement de l'affaire faisait suite à une plainte adressée au Superintendent de Police (SP) par Karam Chand Bhatia, lui aussi membre d'une caste répertoriée mais n'ayant aucun rapport direct avec la victime qui, avant cette affaire, ne l'avait même jamais rencontré.

Avant de détailler le procès, voyons alors comment ce Karam Chand Bhatia a fini par en devenir le plaignant, ce qui me permettra aussi de présenter brièvement le cas tel qu'il m'a été raconté par cet activiste.

\section{Activisme social et médiation judiciaire}

Karam Chand Bhatia est un membre de la caste des Chamar (travailleurs du cuir) et tient une petite boutique de chaussures dans la ville de Shimla, tout près de la District Court.

Il est aussi le leader régional d'un parti politique fondé en 2009, issu d'une faction du parti du Bahujan Samaj Party (BSP), dont l'idéologie défend les droits des Scheduled Castes et des Scheduled Tribes. Dans ce rôle, il prend part à des meetings politiques, développe ses connections locales et nationales, et prépare sa candidature pour les prochaines élections régionales. Mais Karam Chand essaie de tenir son identité politique séparée de ce qu'il définit 
comme son activité "dharmique " ${ }^{10}$, qu'il met en relation avec son rôle de président du Sant Shree Ravi Dass Dharm Saabha, une organisation qu'il a lui-même fondée et qui s'inspire des enseignements du guru Ravidass (XVe siècle), auquel beaucoup de Chamar se réfèrent. C'est en mettant en avant ce rôle dharmique que Karam Chand poursuit ses activités juridiques en aidant les gens des Scheduled Castes à dénoncer les abus commis contre eux par des gens de haute caste. Il les aide à écrire une plainte, ou la rédige à son nom s'ils ont peur des représailles. Dans sa petite boutique, où il vend et répare les chaussures, il entasse aussi d'énormes piles de dossiers contenant différentes sortes de documents judiciaires lettres officielles, copies de plaintes - et nombre d'articles de journaux soigneusement découpés, collés sur des cahiers, datés, concernant les basses castes ou, parfois, des rassemblements politiques. Ce rôle d'intermédiaire entre les pauvres les plus opprimés de la société et le système judiciaire de l'État n'est pas exceptionnel en Inde. D'une part, il est la conséquence du décalage qui existe entre les mesures volontaristes et antidiscriminatoires prises au niveau législatif et l'impossibilité pour des Indiens encore très défavorisés de réclamer leurs droits lorsqu'ils se trouvent en position de subordination ou de dépendance par rapport aux hautes castes. De l'autre, il montre aussi la possibilité, pour des membres de basses castes, de se faire une place publique dans la société, que ce soit en tant qu'intermédiaires juridiques ou comme politiciens.

Ainsi, dans sa petite boutique de chaussures, Karam Chand est très sollicité, non seulement par les basses castes, mais aussi par des voisins du quartier qui n'appartiennent pas à des castes répertoriées mais qui viennent lui demander de l'aide pour rédiger un document adressé au tribunal ou pour savoir comment procéder dans leurs affaires en justice. Tout en ne connaissant ni l'anglais ni le droit (il se fait traduire les actes de loi par un ami anglophone), Karam Chand se dédie avec passion à ce rôle de consultant légal, qui lui a permis de se faire reconnaître même par les juges et les avocats de la ville - lesquels parlent de lui comme d'un social worker ou d'un humanitarian activist. Il est aussi connu des journalistes locaux, grâce auxquels il essaie de faire pression pour que les discriminations contre les Scheduled Castes reçoivent une attention publique.

Ce fut d'ailleurs par la médiation d'un journaliste que Karam Chand fut contacté. Le petit frère de la fillette violée, âgé de 7 ans, avait été d'abord amené au commissariat de police avec l'aide d'un membre de son village. Mais l'officier, vu son jeune âge, lui demanda de revenir avec une plainte écrite et signée par un adulte, pour

10. Le terme vient de dharma, l'ordre cosmique et social (et les règles qui permettent de le préserver). 
qu'il puisse enregistrer l'affaire. Sur le conseil du journaliste, l'adulte qui l'accompagnait prit alors contact avec Karam Chand pour lui demander s'il pouvait l'aider.

En me parlant du cas, Karam Chand me révéla qu'au début il ne voulait pas se mêler de cette histoire. Il savait que les cas de viol sont très difficiles à prouver, car les victimes "acceptent d'abord de parler, puis elles trouvent un compromis avec l'autre partie et à la fin elles nient tout devant le juge. Mais - continua-t-il - lorsque j'ai vu que c'était une toute petite fillette j'ai accepté le dossier..."

Il me raconte ce que le petit frère de la victime lui avait dit: lorsque sa sœur dormait (avec sa famille dans la maison de leur patron Brahmane), Dhiman (l'accusé) « lui bâillonnait la bouche et lui dénouait les cordes du salwar (pantalon indien) pendant qu'elle criait. " Il me dit aussi qu'à l'école la maîtresse avait remarqué des taches de sang sur le pantalon de la fillette; elle lui demanda ce qui s'était passé. D'abord la fillette ne voulut rien dire à la mâ̂tresse, puis elle lui raconta tout et lui dit que «Mamu [oncle] fait ces choses avec moi ${ }^{11}$."

Karam Chand rédigea alors la plainte adressée au Superintendant de Police en donnant davantage de détails : que la mère de la fillette, divorcée, travaillait depuis quatre ans chez le père de l'accusé. Il précisa que « la mère et la victime sont de caste répertoriée Koli" et conclut que "de ce fait, on vous demande d'agir immédiatement et d'enregistrer le FIR contre l'accusé. L'accusé ne doit pas être protégé." "

Le FIR fut enregistré et l'accusé mis en détention préventive. La nouvelle parut dans les journaux sous le titre « Ten-year-old raped for two months: one held" (Hindustan Time, Chandigarh, 2 août 2009).

\section{À la recherche de la victime}

Pendant le procès, lorsque j'ai commencé à suivre le cas en mars 2010, j'ai pu discuter plusieurs fois avec l'Assistant Superintendent of Police (ASP) qui avait mené l'enquête. Il me raconta que lorsqu'il

11. Le cas présenté ici met aussi en évidence la nature des rapports de pouvoir entre famille de haute caste et de basse caste. Tout se passe ici dans un cadre de relative familiarité : la fillette connaît son agresseur, elle l'appelle "mamu» (oncle maternel) ; le viol était commis dans la maison de son patron, dans la chambre où elle dormait avec sa mère et son frère et où l'accusé venait souvent pour regarder la télé avec eux. Ces personnes appartiennent à des castes très différentes mais ont des relations de proximité due à la situation de travail dépendant. Le fait que l'épisode du viol soit enregistré sous le Sc/St Act est par contre la conséquence d'un abus de cette relation de dépendance et de familiarité. 
s'était rendu au village pour commencer les recherches à la suite de la plainte qui avait été déposée, la mère et la fillette avaient disparu: "Nous les avons cherchées pendant toute la nuit en grimpant dans la montagne [...] J'ai même été piqué par des bêtes, c'était vraiment des conditions difficiles. Puis j'ai appelé le père [de l'accusé] et je lui ai dit: "Vous les avez tuées et je vais vous incriminer." Le jour d'après, Anita et sa mère vinrent d'elles-mêmes mais s'entêtèrent à nier. J'ai fait pression sur la mère et je lui ai dit: "Tu es sa maman, en tant que mère comment peux-tu faire comme ça ?" Alors elle m'a tout dit, ce qui s'était passé là-bas. Et j'ai écrit la déposition. »

L'ASP continua : "le rapport médical est clair. Si tu lis ce rapport, tu vas avoir les larmes aux yeux. Cette fillette n'avait que neuf ans! Elle était toute petite et si maigre! Elle n'était pas en très bonne santé." Selon lui, le rapport médical était un élément solide de l'affaire, qui aurait permis au procureur de prouver la culpabilité de l'accusé même si les témoins changeaient leur version devant le tribunal. Il y avait aussi - remarquait-il - le témoignage de Karam Chand (l'auteur de la plainte) et de la maîtresse d'école, " deux témoins crédibles et respectables " qui auraient sans doute, selon lui, confirmé les accusations. Il disait que certains de ses collègues avaient voulu le dissuader d'enregistrer l'affaire car, si les victimes étaient réticentes, elles risquaient sans doute de se rétracter au moment du procès. Mais, disait-il « c'est mon devoir de policier de le faire, et si je ne le fais pas, la fillette continuera à être violée. Je ne fais pas ça pour avoir de la publicité. Je ne connais pas ces personnes et je n'ai rien pris d'eux. Nous avons aussi des enfants à la maison, et s'il leur arrivait ce genre de chose notre cœur serait brisé. "

Les paroles de l'ASP doivent être comprises en tenant compte du reproche, adressé parfois à la police, d'enregistrer (ou de ne pas enregistrer) des affaires à la suite d'une pression externe - que ce soit un intérêt économique, une influence politique ou simplement les liens qu'un policier pourrait avoir avec l'une des parties. On entend aussi dire, surtout dans les milieux des professionnels de la justice, que la police est à la recherche de cas, qu'ils veulent contenter leurs supérieurs, leur montrer qu'ils sont efficaces et obtenir ainsi une promotion. C'est surtout au cours du procès que les policiers sont mis en cause, car ils seront accusés par la défense, au moment de la cross-examination, d'avoir fabriqué l'affaire de toutes pièces. Cela arrive dans des affaires enregistrées directement par la police, où les policiers sont les seuls à confirmer les accusations devant le juge alors que les autres témoins nient en bloc. Dans le cas présenté ici le FIR fait suite à une plainte : ce sera donc le plaignant qui sera d'abord visé par la défense. 


\section{Le procès}

Le récit du viol est confirmé par le plaignant (Karam Chand) au moment où celui-ci est appelé à témoigner, mais contesté par l'avocat de la défense. Dans la transcription écrite de la cross-examination qu'il fait de Karam Chand, on voit tout de suite quelle sera la thèse de l'avocat en regardant la dernière phrase, qu'il dicte au dactylographe sans avoir même posé la question au témoin. Il s'agit en fait d'une phrase de clôture où l'on donne pour certain que ce dernier n'est pas d'accord avec la conclusion que l'avocat propose de l'affaire :

Il est incorrect de suggérer que la présente plainte a été faussement écrite par moi de connivence avec les ennemis du père de l'accusé.

Cette phrase insinue que le cas résulte d'un complot entre les ennemis du père de l'accusé et certains membres du village, qui se seraient servi de l'histoire du viol de la fillette pour mettre en difficulté leur ennemi. La thèse d'un complot à l'origine d'un «faux cas " est systématiquement invoquée par les avocats de la défense et fait partie en Inde de la routine d'un procès. Il arrive que les avocats soient davantage explicites, en désignant des personnes précises - souvent des proches de l'accusé - et même parfois en se servant de témoins pour corroborer leurs théories ${ }^{12}$. Dans le cas en question, l'idée du complot est présentée de façon plus anonyme car elle s'appuie sur l'idée, partagée par tout le monde, de l'existence potentielle d'une tension entre basses castes et hautes castes au niveau local. Le tribunal est donc ici indirectement présenté par l'avocat de la défense comme le lieu où les basses castes, grâce aux lois que l'État a promulguées en leur faveur, parviendraient à se " venger " de leur position de subordination au niveau villageois, à bénéficier d'un rapport de force favorable, ou à pouvoir exercer une pression qu'ils n'avaient pas auparavant ${ }^{13}$.

Dans la transcription du témoignage de la maîtresse d'Anita,

12. Les avocats arrivent aussi parfois à fabriquer des "preuves" de ces histoires fictives de complot, en utilisant des disputes qui sont possibles ou potentielles dans le contexte culturel considéré. Ils essayent de montrer, par exemple, que la vraie dispute entre les parties n'a rien à voir avec la discrimination de caste et que l'affaire n'a été enregistrée sous cet acte que pour transformer une affaire relevant du code civil en affaire criminelle impliquant l'arrestation immédiate de l'accusé.

13. Cette théorie est d'ailleurs en général déjà présentée par l'accusé au début du procès lorsque le juge lui demande s'il se déclare innocent - et donc s'il veut le procès. Ainsi, par exemple : "The accused has taken up a defense of complete denial and claimed that the official and interest witnesses have deposed against him only. He is innocent. He has been falsely implicated by the enemies of his father in the village. " 
entendue par le juge par la suite, l'histoire du viol est racontée à partir de ce qui s'est passé à l'école, lorsque la fillette avait subi un examen médical. À partir des questions qui lui sont adressées au cours de l'audience, la maîtresse, qui est de la même haute caste que l'accusé, raconte qu'un jour à l'école Anita n'était pas bien. Elle était dans un coin et pleurait toute seule. La maîtresse se rapprocha et découvrit des taches de sang sur son salwar. Elle lui demanda ce qui s'était passé. La fillette, après beaucoup d'insistance, lui révéla en pleurant ce qui lui était arrivé, en donnant aussi le nom du garçon. La maîtresse envoya alors la fillette à un "social worker " de l'école qui l'accompagna à l'hôpital pour un contrôle.

Elle appela aussi la mère d'Anita et lorsqu'elle lui révéla ce qu'Anita lui avait dit, la mère commença à pleurer et lui dit que le garçon faisait ça depuis deux ans ${ }^{14}$, qu'elle était absolument impuissante à l'empêcher, et demanda à être aidée.

La maîtresse confirma ainsi devant le juge la version qu'elle avait auparavant donnée à la police : que la fillette lui avait parlé du problème et qu'elle avait révélé l'identité de l'agresseur. De même, la doctoresse confirma pendant l'audience que l'hymen de la fillette était rompu. Voici un extrait de son témoignage :

I medically checked that Anita and found bleeding from her private part. I asked the cause upon which Anita disclosed that one boy named Dhiman had sexually assaulted her due to which she was bleeding. I advised her [the social worker who accompanied Anita to the doctor] to report the matter to the police.

Si les témoignages de la doctoresse, de la maîtresse et de l'activiste allaient tous dans le sens de l'accusation, le problème principal pour le procureur vint de la victime elle-même et de sa mère qui, au moment du procès, nièrent tout ce qu'elles avaient dit auparavant à la maîtresse et à la police. Interrogée par le procureur, Anita dit que ni elle, ni sa mère, ni son frère, n’avaient jamais vécu dans la maison de l'accusé ; qu'elle dormait toujours avec sa mère et son frère dans la même chambre et que l'accusé, qu'elle appelait son mamu (oncle), n'avait jamais été la voir dans sa chambre et ne l'avait jamais agressée.

Ici le juge dicte en anglais la formule utilisée pour introduire une cross-examination :

At this stage, the learned Public Prosecutor has put forth a request that he be allowed to cross-examine the witness because the witness has resiled from her previous statement. Allowed.

14. La presse, les récits des proches, les témoignages lors du procès et les indications dans le texte du jugement ne sont pas uniformes quant à la période durant laquelle les viols ont été répétés, et varient de deux mois à deux ans. 
Dans la cross-examination le juge et le procureur reprennent tout ce qu'Anita avait dit dans le rapport de police, qu'ils lui demandent de confirmer point par point ${ }^{15}$. La fillette répond par la négative à toutes ces questions, mais ces points seront traduits en anglais et retranscrits dans le dossier. Un autre récit émerge alors à travers sa négation même, introduite par "il est inexact que..." "

Voici un passage de la transcription faite au moment du procès :

Il est inexact (it is incorrect) que, ces jours-là, l'accusé Dhiman Sharma entrait et dormait sur le lit de notre chambre et que, dès que ma mère et mon frère s'endormaient, l'accusé me soulevait et me mettait sur lui, puis dénouait le cordon de mon salwar et il mettait ses doigts dans mon vagin, puis son pénis dans mon vagin, et que lorsque je criais l'accusé me couvrait la bouche et me menaçait que si je révélais cela à quelqu'un il me tuerait. Confrontée avec [indications des références du dossier] où c'est ainsi enregistré.

Les accusations se font plus explicites et plus agressives, car il s'agit d'une cross-examination: la question est formulée dans le détail par la personne qui interroge celui ou celle qui est supposé être son propre témoin, mais qui est maintenant dans une attitude de rétractation (hostile). Voici quelques passages de ces interactions transcrites au moment du procès :

Il est inexact que mon frère Hemant m'a aussi vue nue avec l'accusé et qu'après cela l'accusé l'a menacé que s'il allait le révéler à quelqu'un il le tuerait, ni que j’ai affirmé les faits susdits à la police (confronté avec...). Je n'ai jamais dit à Madame Gayatri (la maîtresse) que l'accusé Dhiman commettait ce qui vient d'être affirmé [...] Il est inexact que j'ai révélé cela à la doctoresse [...] Il est inexact que lorsque l'accusé avait un rapport sexuel avec moi mon vagin saignait et que j'ai dit cela à la police.

À la fin de la cross-examination les questions posées par le procureur et par le juge se détachent du rapport de police pour évoquer les raisons pour lesquelles le témoin ne serait pas en train de dire la vérité.

Il est inexact que, par peur, je ne suis pas en train de dire la vérité. Il est inexact que quelqu'un m'a influencée et que de ce fait je suis en train de dire des mensonges. Ma sous-caste est Koli qui fait partie des castes répertoriées. L'accusé Dhiman Sharma est membre d'une caste non répertoriée.

15. Ces phrases sont dictées au greffier directement en anglais et ne sont donc pas comprises par la fillette. Bien qu'elles soient supposées reproduire les questions-réponses qui ont eu lieu au moment du procès, elles reproduisent en fait les phrases telles qu'elles ont été écrites dans le rapport de police. En revanche, les questions posées en hindi à la fillette au moment du procès sont formulées dans un langage plus adapté à un enfant, surtout pour les termes sexuels. 
Avec cette succession de phrases (dictées sans même poser la question, et directement en anglais sans que le témoin comprenne) le juge suggère, au nom du procureur, que la fillette est en train de mentir et qu'elle ment parce que, étant de caste Koli, elle est soumise à la pression de l'accusé qui est de haute caste.

La même théorie est proposée lorsque la mère de la victime est appelée à déposer devant le juge. Il la déclare également (toujours au nom du procureur) hostile witness et, à la fin de la cross-examination, dicte directement au dactylographe une série de phrases prononcées au nom du témoin :

Nous appartenons au même village. Nous nous rencontrons souvent lorsqu'il y a un travail que je dois faire pour eux. Il est inexact de suggérer que maintenant j'ai reçu des compensations (considerations) du père de l'accusé et que je suis en train de faire un faux témoignage afin de sauver l'accusé.

Le procès dura plusieurs semaines ${ }^{16}$, mais dès lors que la fillette et sa mère avaient nié leurs accusations, le procureur savait que l'accusé allait probablement être acquitté. Lorsque les auditions des témoins étaient encore en cours, je suis allée le voir plusieurs fois dans son bureau. C'était un homme plutôt raffiné, passionné de spiritualisme et de méditation, qui aimait bien mettre en avant le contexte social, plutôt que les aspects légaux, des affaires qu'il suivait. C'était lui, d'ailleurs, qui m'avait le premier parlé de cette affaire qui le bouleversait et qui lui tournait dans la tête même pendant la nuit. Il me disait que cela m'intéresserait sûrement et il m'incita plusieurs fois à aller voir sur place ce qui se passait là-bas, au village, pour que j'essaye de comprendre pourquoi la fillette et la maman étaient devenues hostile. Il était encore incrédule: comment avaient-elles pu tout nier devant le juge? " Oui, disait-il, j'aurais pu les dénoncer d'avoir menti devant le tribunal. Mais pour quoi faire ? Elle a été violée et en plus elle va être punie pour avoir menti ? Qu'est-ce que l'on obtient avec ça ? "Il s'en prenait alors à la société, au problème de la pauvreté et de la domination des hommes sur les femmes. Mais il s'en prenait aussi à l'État, dont le devoir, disait-il, était de protéger les témoins. "Elle [la maman] aurait dû être éloignée de la compagnie du père de l'accusé et on aurait dû lui offrir un emploi quelque part ailleurs. Autrement qu'est-ce que M. Kochhar [lui même] et M. Gupta [le juge] pouvaient faire ? [...] Parfois, vous savez, quand vous êtes au tribunal et vous entendez mentir, mentir, mentir... ça vous rend fou! Une seule phrase de la mère aurait pu suffire dans cette affaire. Mais, même maintenant, la mère de la fillette continue à vivre avec le

16. Lorsque j'ai commencé à suivre les audiences, le procès était déjà en cours. 
père de l'accusé, elle a des relations avec lui ! C'est pour ça qu'elle s'est rétractée. "

Il était aussi furieux de ce qui s'était passé au moment de l'audience :

Lorsque la fillette était devant le juge, je lui ai demandé de dire tout ce qu'elle avait dit à la police. Elle s'est tournée vers moi pour me parler et à ce moment-là l'avocat l'a tirée par derrière. Alors la fillette a dit: "il ne m'est rien arrivé. " J'étais en colère et j'ai dit à l'avocat "qu'est-ce que c'est que ça ? Pourquoi tu l'as tirée par derrière ?" [...] J'étais vraiment dégoûté. J'avais tout essayé pour la faire parler, je lui avais même montré des photos pour qu'elle choisisse celui qui lui avait fait ça. Si la maman ne s'était pas rétractée, je ne l'aurais pas appelée à témoigner. Elle n'avait que 9 ans... Mais puisque la mère faisait comme ça, j'ai décidé de l'interroger [la fillette] avec l'espoir qu'elle puisse dire quelque chose.

Quoique découragé, il disait ne pas avoir renoncé à gagner cette affaire. Il aurait maintenant défendu le cas sous un autre angle. « Pourquoi la maîtresse aurait-elle menti ? - disait-il - Quelle était son animosité envers l'accusé ? Il n'y avait aucune animosité. Il me dira [le juge] que c'est un hearsay [ouï-dire], que c'est une circumstantial evidence [preuve indirecte]. Mais moi je dirai qu'il y a plus que cela. Ils ont vu du sang sur le tapis, là où seule la fillette était assise. Il y a aussi le rapport médical. Pourquoi la doctoresse aurait-elle dû soutenir l'accusation?"

De fait, au moment de la plaidoirie (arguments), le juge observa que, puisque la fillette et sa mère étaient devenues hostile, les trois témoignages qui auraient pu servir à corroborer l'accusation (celui de la maîtresse, de l'activiste et de la doctoresse) étaient désormais dépourvus de valeur : ils étaient devenus des ouï-dire.

Parmi les interactions qui eurent lieu au moment des arguments, le juge, tout en faisant comprendre qu'il aurait acquitté l'accusé, posa à l'avocat une question qui montre l'importance donnée à la dimension sociale de l'affaire. "Que dit la société ? Que disent les médias?", demanda-t-il. L'avocat répondit qu'au moment de l'enquête il y avait eu des articles de presse et qu'il y avait eu beaucoup de pression médiatique, mais que maintenant tout était calme. Il ajouta aussi que "d'ailleurs, s'il faut décider sur la base des médias, ce n'est pas la peine de venir au tribunal!"

Le procureur reprit alors la parole en mettant en avant le rapport médical qui montrait que la fillette "avait été soumise à un rapport sexuel » et qu'elle saignait au moment du contrôle. L'avocat argumenta que le sang pouvait être dû à "n'importe quelle blessure qu'elle aurait pu se faire en jouant avec un objet extérieur " - une idée qui ressort constamment dans ce genre d'affaire. Bien que le juge n'adopte pas dans son jugement l'hypothèse de l'avocat, il écrivit que « le procureur était tenu de prouver les faits 
avancés [qu'elle avait été soumise à un rapport sexuel par l'accusé] sur la base du témoignage de la victime ou d'un autre témoin. " À la fin des arguments, devant les protestations du procureur, insistant que la justice devait être rendue indépendamment des négociations privées entre les parties, le juge répondit, résigné " Qu'estce qu'on peut faire maintenant ? La justice... peut seulement être faite par Dieu."

Dans son jugement d'une quinzaine de pages, le juge conclut que :

Nous regrettons de constater que la victime et sa mère ont tourné le dos aux positions de l'accusation et n'ont en rien soutenu celle-ci.

Il s'appuya sur plusieurs décisions, certaines très anciennes, que l'avocat avait citées au cours des arguments et qui concernaient l'admissibilité des preuves. Il en conclut que :

Confrontée aux preuves discutées, aux faits enregistrés et aux lois citées ci-dessus, la cour est laissée sans autre choix que d'affirmer et conclure que l'accusation a totalement failli à prouver sa position audelà de tout doute raisonnable.

Pourtant, lors de mes conversations avec le juge, celui-ci ne semblait avoir aucun doute sur le fait que la fillette avait été régulièrement violée par le garçon. Il savait aussi que la mère de la fillette continuait à vivre chez le père de l'accusé et qu'elle avait maintenant reçu, comme lui-même me le dit, des compensations. C'est d'ailleurs ce qu'il avait suggéré lors de la cross-examination pendant laquelle, tout en parlant au nom du procureur, il avait dicté plusieurs formules indiquant les raisons qui avaient poussé la mère de la fillette à nier les accusations. Mais, le juge m'expliqua par la suite :

D’un point de vue légal, c'est différent. Imaginez que vous disiez que quelqu'un a donné une gifle à un autre et veniez immédiatement me le dire. Votre témoignage, même s'il est indirect, sera pertinent car il pourra corroborer le témoignage de la victime. Mais supposez que la personne frappée vienne me dire qu'elle n'a reçu aucune gifle de personne; alors votre témoignage n'est plus pertinent, il devient de deuxième main. Vous savez, c'est très difficile d'être juge dans ces cas...

À propos de l'accusé il me dit aussi

[...] n'avoir aucune sympathie pour lui [...] mais même si je pense que c'est un criminel, je ne peux rien faire, je suis étroitement lié [...] Nous ne pouvons pas ignorer la loi de l'État. Même si je suis de l'avis que les juges devraient être laissés libres de décider le cas de plusieurs façons.

$\mathrm{Si}$, en dehors du tribunal ou lors des evidence, la position du juge et celle du procureur étaient proches - tous les deux étaient con- 
vaincus que la fillette et sa mère mentaient - au moment de la plaidoirie et du jugement leurs positions divergeaient quant à la valeur légale des autres témoignages : le juge estimait que cela relevait du ouï-dire tandis que le procureur mettait en avant leur crédibilité. Cette divergence, que j'avais remarquée au moment du procès, s'est néanmoins atténuée avec le temps. Presqu'un an après le jugement, lorsque je suis retournée sur le terrain, j'ai à nouveau rendu visite au procureur et lui ai demandé si l'affaire avait été envoyée en appel. Il me dit que ce cas n'était pas approprié pour un appel car le rapport médical n'était finalement pas très clair et qu'il n'aurait pas été suffisant pour faire condamner l'accusé. Il demanda alors à son assistant d'aller chercher le dossier pour me montrer le rapport qu'il avait fait au gouvernement, car il incombe au procureur de lui envoyer le dossier, avec son avis sur la nécessité d'un réexamen de l'affaire par la Haute Cour de l'État (High Court) ${ }^{17}$. Mais une chose inexplicable à ses yeux s'était produite : le dossier avait disparu ; il n'avait pas été envoyé au gouvernement et aucune sortie n'était marquée sur le registre. Pendant plusieurs jours, les assistants du procureur cherchèrent le dossier, sans succès. Des rumeurs commencèrent à circuler sur un autre procureur, qui aurait fait disparaître le dossier à la demande du père de l'accusé pour être sûr que l'affaire ne soit pas renvoyée en appel. Le procureur me dit qu'il ouvrirait une enquête, mais jusqu'à présent le dossier n'a pas été retrouvé.

\section{Le récit de l'affaire dans la localité}

La connaissance du contexte social local que les professionnels impliqués dans l'affaire (police, procureur, juge) démontrent lorsqu'ils en discutent en dehors de la salle d'audience, tout en étant plus large que ce qui ressort des interactions traduites et verbalisées au moment du procès, fournit l'image d'une communauté villageoise complètement dominée par les rapports de force locaux. $\mathrm{Au}$ village de la victime, néanmoins, l'histoire entre la fillette et son agresseur, racontée par l'une de ses protagonistes, prend une autre profondeur. Elle montre non seulement des personnages plus nuancés que ceux qui apparaissent devant le juge, mais aussi que l'affaire, telle qu'elle ressort des investigations et du tribunal, n'est que l'acte final d'une histoire dont le début est plus ancien et dont certaines dynamiques sont complètement passées sous silence devant les représentants de la justice officielle.

Presqu'un an après la fin du procès, la maîtresse d'école révéla à mon assistant qu'au départ, lorsque la fillette lui raconta ce qui

17. L'année d'avant, je n'avais pas pu voir son rapport car j'avais dû quitter le terrain avant que le procureur ne le rédige. 
s'était passé, sa réaction avait été d'essayer d'arranger les choses avec la famille de l'agresseur. Voici quelques passages de son récit.

Elle [Anita] n'était pas bien et au début la maman m'avait dit qu'elle avait des calculs. Je l'ai crue et je lui ai même suggéré de suivre un traitement et de se faire aider par eux (par la famille où elle travaillait) [...] Mais la fillette allait de pire en pire. Elle était toujours malade, ne faisait plus ses devoirs, ne mangeait pas, elle restait tout le temps dans un coin à pleurer. Je l'ai appelée dans mon bureau et j'ai insisté pour qu'elle me dise ce qu'elle avait. Elle me raconta alors ce qui s'était passé mais me dit qu'on lui avait interdit de parler [...] Elle avait une telle peur [...] Elle disait que la mère du garçon avait menacé de lui couper la tête si elle parlait. Elle m'a dit alors "Madame, prenezmoi chez vous sinon elle [la mère du jeune homme] va me tuer. »

Du coup j'ai tout compris. J'ai aussi compris pourquoi les parents du garçon avaient commencé à m'envoyer plein de légumes et de fruits depuis quelque temps. Ils m'envoyaient beaucoup de légumes et je ne comprenais pas pourquoi.

Les signes du problème d'Anita s'étaient donc manifestés avant que la maîtresse d'école découvre les saignements et qu'elle envoie la fillette à l'hôpital. Rester dans un coin à pleurer, ne pas manger, ne pas faire les devoirs étaient les seuls moyens dont disposait Anita pour exprimer son malheur devant la maîtresse tout en ne pouvant pas parler. L'école représentait pour elle désormais le seul cadre où elle se sentait protégée, où elle pouvait attirer l'attention pour que quelqu'un l'aide à dire ce qu'elle devait taire. On voit aussi qu'à ce stade les parents du garçon, qui étaient au courant de ce que celui-ci faisait au point qu'ils avaient menacé la fillette de la tuer si elle disait quelque chose, commençaient à craindre que la maîtresse fût mise au courant de l'affaire. Profitant peut-être aussi des liens de caste entre celle-ci et la famille du garçon (tous deux étant Brahmanes), ils envoyèrent des " cadeaux " pour créer ce que la maitresse appelait " de bonnes relations " qui l'auraient prédisposée à ne pas croire à ce que la fillette (provenant d'une Scheduled Caste) aurait pu dire contre eux. Mais ensuite, une fois les saignements évidents, la maîtresse parvint à convaincre Anita de tout lui dire, ce qui aurait déclenché - selon la version du procès - la prise en charge officielle du problème (contrôle médical, plainte, entrée en scène de la police, etc.).

En fait, les révélations d'Anita n'avaient pas du tout, dans un premier temps, conduit la maîtresse à saisir la justice de l'État. Voici comment elle continue son récit :

Le père du garçon avait admis que ce que son fils faisait n'était pas bien, mais il me pria de ne pas appeler la police : il aurait fait tout ce que je lui aurais demandé. Il utilisa quelqu'un du personnel de l'école pour m'offrir de l'argent afin que je ne dise rien à la police. Je lui ai alors demandé de verser 400000 roupies sur un compte au nom de la fillette et de mettre de la terre à son nom. Désormais la fillette était 
finie $^{18}$, elle était dans un état pitoyable. Il me dit alors qu'il était d'accord, qu'il allait faire ça mais que je ne devais pas appeler la police. [...] Mais la police commença à venir au village car quelqu'un leur avait dit quelque chose [...] [Les policiers] nous créaient beaucoup de problèmes et me disaient "Madame, pourquoi vous ne voulez pas témoigner?" Mais nous n'avons rien dit, nous ne voulions pas aider la police à ce moment-là. S'il pouvait y avoir un compromis, cela aurait été bien parce qu'au tribunal l'affaire va traîner, traîner...

Même lorsqu'Anita eut tout dit à la maîtresse, celle-ci avait décidé que l'affaire devait se résoudre localement. Le fait que l'accusé était quelqu'un du village, et de la famille de laquelle la mère de la fillette dépendait pour sa survie, avait conduit la maîtresse à préférer la voie du compromis plutôt que celle de la justice d'État. L'hostilité même qu'elle montrait vis-à-vis des officiers de police qui leur "créaient beaucoup de problèmes", et auxquels elle ne voulait rien dire, viendrait confirmer la théorie de "l’État étranger ", de l'existence d'une opposition entre la justice d'État et les pratiques de résolution villageoise des conflits. Mais les choses changèrent brusquement, comme elle-même le raconte :

Puis un jour, je passais à côté de leur maison [du père du garçon] et je lui dis alors que j’allais appeler le pradhan [président du village] pour qu'il puisse effectuer le transfert d'argent et de terre au nom de la fillette, et vous savez ce qui s'est passé ? La mère de la fillette, qui était à côté de lui, me dit: "Qu'est-ce que vous dites, madame ji, pourquoi nous devrions prendre de l'argent de lui ? Pourquoi êtes-vous en train de l'insulter? " Alors je n'ai plus rien voulu savoir. Vous vous rendez compte? C'était sa mère ! Et ce qu'elle m'avait dit lorsqu'elle était venue à l'école était complètement différent. Elle m'avait dit de l'aider, qu'elle ne savait pas comment se sortir de cette situation, qu'elle avait besoin du travail qu'elle faisait chez eux car elle avait deux enfants à nourrir. Elle était même venue me voir en cachette car on lui avait interdit de venir à l'école. J'avais essayé de l'aider et maintenant elle me parlait comme ça! J'étais tellement furieuse que si j'avais été plus proche d'elle je lui aurais cassé la tête. Alors je leur ai dit que j'allais appeler la police.

Le fait que le crime n'allait pas être réparé par un compromis et qu'il était même nié par la mère de la victime amène la maîtresse à changer d'idée : c'était maintenant l'État qui devait intervenir, non seulement pour indemniser la fillette, mais aussi pour punir le coupable. Ce changement fut provoqué par l'attitude de la mère, qui s'était finalement laissée dominer par son patron. Elle avait pourtant montré qu'elle aussi cherchait à réagir à sa position soumise lorsqu'elle était allée voir la maîtresse en cachette pour lui demander de l'aider. C'est lorsque les méthodes locales de compen-

18. L'idée exprimée par la maîtresse est que lorsqu'une fille a été violée elle ne pourra plus se marier. 
sation ne furent pas mises en œuvre et que les rapports de domination prirent le dessus que la maîtresse adopta le système de justice de l'État. Du coup, l'écart s'élargit entre des stratégies locales de domination et la logique de la loi. Mais les positions et les réactions de la famille de la victime diffèrent, comme le montre la suite du récit de la maîtresse :

Depuis ce jour ils interdirent à la fillette de venir à l'école. Ils avaient peur que la police n'arrive et ne prenne sa déposition. Puis j'ai reçu un appel du pradhan qui me dit « la police est ici, ils veulent interroger la fillette », mais je lui ai dit que depuis des jours elle n'était pas là, qu'il n'y avait que son petit frère. Puis, avec ma collègue, nous avons eu l'idée de demander au garçon s'il savait ce qui était arrivé à sa sœur. Et vous savez quoi? Il nous a tout dit ! Et quel tableau horrible il nous a donné ! C'était incroyable! Alors j'ai tout de suite rappelé le pradhan pour lui dire : "Vite, le garçon sait tout, il faut l'amener à la police pour qu'il puisse témoigner. " Le pradhan est venu, il a pris le petit garçon dans ses bras et l'a amené à la police. Et lui leur a tout dit. Nous étions vraiment contents de voir quel courage il avait, ce petit garçon.

L'enfant n'avait que sept ans mais, n'étant pas la victime directe, il avait dû se sentir moins directement menacé que sa sœur par rapport à l'interdiction de parler. C'est finalement grâce à lui que des passerelles entre la justice officielle et la localité ont pu être établies. Il racontera tout, d'abord à la police et puis à Karam Chand, l'activiste de Scheduled Caste qui, vu le jeune âge du garçon, se chargera finalement de déposer la plainte officielle. Néanmoins, sa parole ne fut pas prise en compte par le procureur qui ne l'inclut pas dans la liste des témoins car il pensait que, comme sa sœur, lui aussi aurait été forcé de tout nier devant le juge.

Maintenant que le procès était terminé, que l'accusé avait été acquitté et était rentré au village, la maîtresse n'était pas du tout contente de la façon dont les choses s'étaient terminées. Elle révéla que le père du garçon venait juste d'organiser une grande cérémonie chez lui en honneur de la divinité du village pour la remercier, aux yeux de tout le monde, d'avoir aidé son fils à être acquitté. Quant à elle, elle avait désormais peur de rencontrer les gens de la famille qui la regardaient pleins de colère.

C'est très dangereux pour nous. Vous savez par exemple ce que le garçon a fait une fois sorti de prison ? Il est venu ici pendant les vacances et la nuit il nous a cassé les toilettes de l'école. On venait de les refaire et il les a complètement cassées avec de grosses pierres.

Bien que le père de Dhiman ait maintenant construit une petite maison pour la mère de la fillette et ses deux enfants, elle trouvait que justice n'était pas faite, et que le jeune homme aurait dû rester en prison beaucoup plus longtemps. 
Le juge aurait dû rendre son jugement sur la base du rapport médical. Ça ne suffit pas de dire qu'on ne peut rien faire. Est-ce que la doctoresse s'était trompée alors? Et si elle s'était trompée, il aurait fallu prendre des mesures contre elle. Ou il aurait fallu à nouveau faire un contrôle médical. Ça ne peut pas finir comme ça! Le juge aurait dû juger sur la base de ce que la doctoresse a dit et si la mère s'est rétractée il aurait dû comprendre pourquoi. [...] Nous avons essayé de donner ses droits à cette fillette...

Ayant opté pour la justice de l'État, la maîtresse d'école protestait contre la décision du juge et adoptait le langage du droit. Elle aurait pu être satisfaite du fait que le père avait à la fin fait construire une maison pour Anita et sa famille (en plus de l'argent qu'il pouvait leur avoir donné). Mais ce n'était pas cela le "compromis" qu'elle avait initialement souhaité, celui qui aurait dû être fait devant le pradhan et en reconnaissant le crime devant la communauté villageoise. Ce qu'elle n'arrivait pas à accepter, c'est que le procès avait publiquement établi que l'offense n'avait pas eu lieu, et que sa parole, celle de Karam Chand et celle de la doctoresse n'avaient pu suffire à faire condamner l'accusé.

\section{Conclusion}

Les tribunaux indiens ont été souvent définis dans les travaux anthropologiques comme des institutions aveugles au contexte local et aux réseaux des relations sociales dans lesquelles une personne se trouve impliquée. Nitya Rao, par exemple, note comment dans les tribunaux du Jharkhand «le contexte local est complètement ignoré, ce qui est privilégié est la notion de droits de l'homme." Il remarque aussi que "l'exclusion du discours local fait que les décisions prises ici [au tribunal] sont difficilement appliquées sur le terrain " (2007: 316). Ces idées sont souvent la conséquence d'une approche des tribunaux qui, pendant longtemps, a eu pour souci principal de les opposer aux conseils de village, les panchâyat, ou à d'autres formes d'instances décisionnaires au niveau villageois (Cohn 1959 ; Hayden 1984 ; Moore 1993). Elles correspondent aussi à l'image que certains juges veulent donner d'euxmêmes, comme arbitres neutres dont les décisions reposeraient uniquement sur les "faits enregistrés " et sur les règles de la preuve (rules of evidence).

Les données ethnographiques qui ont été ici présentées montrent néanmoins comment les dynamiques sociales dans lesquelles s'inscrit une affaire judiciaire sont un sujet régulier de conversation parmi les professionnels en charge du cas. Elles sont aussi utilisées au moment du procès en tant qu'arguments pour suggérer la raison pour laquelle des témoins sont soupçonnés de ne pas dire la vérité. Ici, en particulier, elles ont été évoquées par le juge et par le procureur à propos de la mère de la victime, pour suggérer 
qu'elle avait reçu des compensations de la part du père de l'accusé, ou pour souligner la dépendance économique qui la lie à la famille de celui-ci. Elles ont été évoquées aussi par l'avocat de la défense, pour formuler sa théorie du complot. Ces bribes de référence au contexte sociologique au moment du procès assument souvent une valeur stratégique, dont le but est de décrédibiliser un témoin ou de suggérer pourquoi il est devenu " hostile", dans le cadre de formules d'écriture judiciaire. Elles semblent fournir des explications conventionnelles, davantage destinées à être prises en compte par les juges qu'à contribuer à la recherche d'une vérité, car elles sont écrites tout à la fin d'une transcription, comme phrases conclusives de la cross-examination, et parfois directement en anglais.

Même si la décision du juge et le rapport du procureur constituent en définitive une reconnaissance explicite du fait que les négociations privées entre les parties ont prévalu sur la justice de l'État, ce qui ressort des audiences - et surtout ce qui est verbalisé - montre néanmoins comment autant le juge que le procureur mettent différemment l'accent, selon la phase du procès, sur une compréhension de l'affaire en termes sociologiques ou sur une compréhension en termes légaux.

Paradoxalement, ce sont les victimes elles-mêmes, en se rétractant, qui ont nié toute influence des dynamiques sociales au moment du procès. Mais il serait inexact de présenter leur rétractation comme une simple démonstration de l'opposition entre système judiciaire et système villageois. L'exemple présenté ici montre comment, dans le cas des villageois, les mêmes personnes peuvent, selon les moments ou les circonstances, s'inscrire dans la reconduction des rapports de force locaux ou, au contraire, adopter la logique de la loi. Nous avons vu comment Anita, bien que terrorisée par les menaces, était arrivée à attirer l'attention du personnel de l'école et à raconter ce qui s'était passé. La mère également, tout en étant à la fin complètement contrôlée par son landlord, avait cherché à un moment à s'en sortir en se cachant même pour aller rencontrer la maîtresse et lui demander de l'aider.

Si dans les cas de la fillette et de la mère le passage entre subordination et dénonciation est ambigu et dépend de leur capacité à résister aux pressions et aux menaces provenant de leurs patrons, dans le cas de la maîtresse ce changement se manifeste de façon plus tranchée et résulte de l'impasse dans laquelle les procédures locales de compensation semblaient être tombées. Le personnage de Karam Chand, quant à lui, montre de quelle manière, dans ce même milieu de castes économiquement défavorisées et socialement subordonnées, certains individus ont complètement abandonné les instances villageoises de résolution des conflits et se mobilisent pour la mise en pratique des principes d'égalité énoncés par la loi. 
Il serait réducteur d'interpréter ces changements d'attitude de la part des protagonistes uniquement en termes de manipulations ou de résistances. Une approche centrée sur les acteurs (Lewis 1993), prenant en compte non seulement les interactions officielles du procès mais aussi les récits individuels des différents protagonistes, permet de mieux restituer cette dimension subjective du rapport qu'ont les villageois avec les procédures judiciaires étatiques. Elle montre aussi comment une approche des pratiques de justice qui se préoccuperait uniquement d'analyser, voire de dénoncer, l'origine coloniale des catégories ou des procédures judiciaires, ou la façon dont celles-ci continuent à reproduire des rapports de pouvoir ou de subordination, tout en étant pertinente d'un point de vue historique, ne suffirait pas à restituer la multiplicité et la variabilité des attitudes et des comportements des acteurs concernés, leurs convictions, leurs passions ou leurs hésitations.

Daniela BERTI. (CEH-CNRS.)

\section{Références}

Anderson M. R. (1990) "Classifications and Coercions: Themes in South Asian Legal Studies in the 1980s ", South Asia Research 10(2) : 158177.

Baxi U. (2003) "The Colonialist Heritage", dans P. L. Munday et R. Munday (éds), Comparative Legal Studies: Traditions and Transitions, p. 46-75. Cambridge : Cambridge University Press.

Berti D. (2010) « Hostile Witnesses, Judicial Interactions and Out-ofCourt Narratives in a North Indian District Court ", Contributions to Indian Sociology 44(3) : 235-263.

Berti D. (2011a) «Trials, Witnesses and Local Stakes in a District Court of Himachal Pradesh (North India) ", dans J. Pfaff et G. Toffin (éds) Citizenship, Democracy, and Belonging in the Himalayas, p. 290-313. New Delhi : Sage.

Berti D. (2011b) «Courts of Law and Legal Practice», dans I. ClarkDeces (éd.) A Companion to the Anthropology of India, p. 355-370. Oxford: Blackwell.

Brown M. (2003) «Ethnology and Colonial Administration in Nineteenth-Century British India: the question of native crime and criminality ", British Journal for the History of Science 36(2) : 201-219.

Cohn B. S. (1959) « Some Notes on Law and Change in North India", Economic Development and Cultural Change 8(1) : 79-93.

Cohn B. S. (1965) « Anthropological Notes on Disputes and Law in India ", American Anthropologist, 67(6), The Ethnography of Law : 82-122.

Davies M., Croall H. et Tyrer J. (1995) Criminal Justice: An Introduction to the Criminal Justice System in England and Wales. London: Longman.

Eckert J. (2006) « From Subjects to Citizens: Legalism from Below and the Homogenisation of the Legal Sphere ", Journal of Legal Pluralism 53$54: 45-75$. 
Galanter M. L. (1992) "The Aborted Restoration of "Indigenous" Law in India ", dans M. L. Galanter, Law and Society in Modern India (introduction by R. Dhavan), p. 37-53. Delhi : Oxford University Press.

Guha R. (1989) "Dominance without Hegemony and its Historiography ", Subaltern Studies VI : Writings on South Asian History and Society, p. 210-309. Oxford : Oxford University Press.

Hayden R. (1984) «A Note on Caste Panchâyats and Government Courts in India: Different Kinds of Stages for Different Kinds of Performances ", Journal of Legal Pluralism, $22: 43-52$.

Kolsky E. (2005) "Codification and the Rule of Colonial Difference: Criminal Procedure in British India ", Law and History Review 23(3) : 631-683.

Lewis H. S. (1993) "A New Look at Actor-Oriented Theory", PoLAR (Political and Legal Anthropology Review) 16(3) : 49-56.

Ludden D., éd. (2001) Reading Subaltern Studies: Critical History, Contested Meaning, and the Globalisation of South Asia. New Delhi : Permanent Black.

Merry S. E. (2003) «Rights Talk and the Experience of Law: Implementing Women's Human Rights to Protection from Violence ", Human Rights Quarterly 25(2) : 343-381.

Moore E. P. (1993) «Gender, Power, and Legal Pluralism: Rajasthan, India ", American Ethnologist 20(3) : 522-542.

Moore H. (1994) "The Problem of Explaining Violence in the Social Sciences ", dans P. Harvey et P. Gow (éds) Sex and Violence: Issues in Representation and Experience, p. 522-542. London : Routledge.

Rao N. (2007) "Custom and the Courts: Ensuring Women's Rights to Land, Jharkhand, India ", Development and Change 38(2) : 299-319.

Roussel G. (2005) Les procès-verbaux d'interrogatoire : Rédaction et exploitation. Paris : L'Harmattan.

Shah A. M. (1998) The Family in India: Critical Essays. New Delhi : Orient Longman.

Sivaramakrishnan K. (2008) "Some Intellectual Genealogies for the Concept of Everyday Resistance ", American Anthropologist 107(3) : 346355 .

Srinivas M. N. (1962) Caste in Modern India, and Other Essays. Mumbai : Asia Publishing House.

Stein B. (1980) Peasant, State and Society in Medieval India. Delhi : Oxford University Press.

Wardhaugh J. (2005) "The Jungle and the Village: Discourses on Crime and Deviance in Rural North India ", South Asia Research 25(2) : 129140. 